\title{
Lacunary Statistical Limit Points in Random 2-Normed Spaces
}

\author{
A. Güncan, U. Yamanc1, and M. Gürdal \\ Department of Mathematics, Suleyman Demirel University, 32260 Isparta, Turkey \\ Correspondence should be addressed to M. Gürdal; gurdalmehmet@sdu.edu.tr
}

Received 29 April 2013; Accepted 13 June 2013

Academic Editors: F. Colombini and J.-L. Wu

Copyright (C) 2013 A. Güncan et al. This is an open access article distributed under the Creative Commons Attribution License, which permits unrestricted use, distribution, and reproduction in any medium, provided the original work is properly cited.

We introduce the notion $\theta$-cluster points, investigate the relation between $\theta$-cluster points and limit points of sequences in the topology induced by random 2-normed spaces, and prove some important results.

\section{Introduction and Background}

An interesting and important generalization of the notion of metric space was introduced by Menger [1] under the name of statistical metric space, which is now called probabilistic metric space. In this theory, the notion of distance has a probabilistic nature. Namely, the distance between two points $x$ and $y$ is represented by a distribution function $F_{x y}$; and for $t>0$, the value $F_{x y}(t)$ is interpreted as the probability that the distance from $x$ to $y$ is less than $t$. In fact the probabilistic theory has become an area of active research for the last forty years. An important family of probabilistic metric spaces are probabilistic normed spaces. The notion of probabilistic normed spaces was introduced in [2] and further it was extended to random/probabilistic 2-normed spaces by Goleţ [3] using the concept of 2-norm of Gähler [4]. Applications of this concept have been investigated by various authors, for example, [5-7].

The concept of statistical convergence for sequences of real number was introduced by Fast in [8] and Steinhaus in [9] independently in the same year 1951. A lot of developments have been made in this area after the works of Salat [10] and Fridy [11]. Recently, Mohiuddine and Aiyub [12] studied lacunary statistical convergence as generalization of the statistical convergence and introduced the concept $\theta$ statistical convergence in random 2-normed space. In [13], Mursaleen and Mohiuddine extended the idea of lacunary statistical convergence with respect to the intuitionistic fuzzy normed space. Also lacunary statistically convergent double sequences in probabilistic normed space was studied by Mohiuddine and Savaş in [14].
The aim of this work is to introduce and investigate the relation between $\theta$-statistical cluster points, $\theta$-statistical limit points, and ordinary limit points of sequence in random 2-normed spaces.

First, we recall some of the basic concepts that will be used in this paper. All the concepts listed below are studied in depth in the fundamental book by Schweizer and Sklar [2].

Let $\mathbb{R}$ denote the set of real numbers and $\mathbb{R}_{+}=\{x \in \mathbb{R}$ : $x \geq 0$. A mapping $f: \mathbb{R} \rightarrow \mathbb{R}_{+}$is called a distribution function if it is nondecreasing and left continuous with $\inf _{t \in \mathbb{R}} f(t)=0$ and $\sup _{t \in \mathbb{R}} f(t)=1$.

We denote the set of all distribution functions by $D^{+}$such that $f(0)=0$. If $a \in \mathbb{R}_{+}$, then $H_{a} \in D^{+}$, where

$$
H_{a}(t)= \begin{cases}1, & \text { if } t>a \\ 0, & \text { if } t \leq a\end{cases}
$$

It is obvious that $H_{0} \geq f$ for all $f \in D^{+}$.

A triangular norm ( $t$-norm) is a continuous mapping $*:[0,1] \times[0,1] \rightarrow[0,1]$ such that $([0,1], *)$ is an abelian monoid with unit one and $c * d \leq a * b$ if $c \leq a$ and $d \leq b$ for all $a, b, c, d \in[0,1]$. A triangle function $\tau$ is a binary operation on $D^{+}$which is commutative, associative and $\tau\left(f, H_{0}\right)=f$ for every $f \in D^{+}$.

The concept of 2-normed spaces was first introduced by Gähler $[4,15]$.

Let $X$ be a real vector space of dimension $d$, where $2 \leq$ $d<\infty$. A 2-norm on $X$ is a function $\|\cdot, \cdot\|: X \times X \rightarrow \mathbb{R}$ which satisfies (i) $\|x, y\|=0$ if and only if $x$ and $y$ are linearly 
dependent; (ii) $\|x, y\|=\|y, x\|$; (iii) $\|\alpha x, y\|=|\alpha|\|x, y\|, \alpha \in$ $\mathbb{R}$; (iv) $\|x, y+z\| \leq\|x, y\|+\|x, z\|$. The pair $(X,\|\cdot, \cdot\|)$ is then called a 2 -normed space.

As an example of a 2-normed space we may take $X=\mathbb{R}^{2}$ being equipped with the 2-norm $\|x, y\|:=$ the area of the parallelogram spanned by the vectors $x$ and $y$, which may be given explicitly by the formula

$$
\|x, y\|=\left|x_{1} y_{2}-x_{2} y_{1}\right|, \quad x=\left(x_{1}, x_{2}\right), y=\left(y_{1}, y_{2}\right) \text {. }
$$

In 2006, Goleţ [3] introduced the notion of random 2-normed space.

Let $X$ be a linear space of dimension greater than one, $\tau$ a triangle, and $F: X \times X \rightarrow D^{+}$. Then $F$ is called a probabilistic 2 -norm and $(X, F, \tau)$ a probabilistic 2 -normed space if the following conditions are satisfied:

(i) $F(x, y ; t)=H_{0}(t)$ if $x$ and $y$ are linearly dependent, where $F(x, y ; t)$ denotes the value of $F(x, y)$ at $t \in \mathbb{R}$,

(ii) $F(x, y ; t) \neq H_{0}(t)$ if $x$ and $y$ are linearly independent,

(iii) $F(x, y ; t)=F(y, x ; t)$ for all $x, y \in X$,

(iv) $F(\alpha x, y ; t)=F(x, y ; t /|\alpha|)$ for every $t>0, \alpha \neq 0$ and $x, y \in X$,

(v) $F(x+y, z ; t) \geq \tau(F(x, z ; t), F(y, z ; t))$ whenever $x, y$, $z \in X$.

If $(\mathrm{v})$ is replaced by

$(\mathrm{v})^{\prime} F\left(x+y, z ; t_{1}+t_{2}\right) \geq F\left(x, z ; t_{1}\right) * F\left(y, z ; t_{2}\right)$ for all $x, y, z \in X$ and $t_{1}, t_{2} \in \mathbb{R}_{+}$, then $(X, F, *)$ is called a random 2-normed space (for short, RTN space).

Remark 1 . Note that every 2 -normed space $(X,\|\cdot, \cdot\|)$ can be made a random 2-normed space in a natural way, by setting

$$
F(x, y ; t)=H_{0}(t-\|x, y\|),
$$

for every $x, y \in X, t>0$ and $a * b=\min \{a, b\}, a, b \in[0,1]$.

Let $(X, F, *)$ be a RTN space. Since $*$ is a continuous $t$-norm, the system of $(\varepsilon, \lambda)$-neighborhoods of $\theta$ (the null vector in $X$ )

$$
\left\{\mathcal{N}_{\theta, z}(\varepsilon, \eta): \varepsilon>0, \eta \in(0,1), z \in X\right\},
$$

where

$$
\mathcal{N}_{\theta, z}(\varepsilon, \eta)=\left\{x, z \in X \times X: F_{x, z}(\varepsilon)>1-\eta\right\}
$$

determines a first countable Hausdorff topology on $X \times X$, called the F-topology. Thus, the F-topology can be completely specified by means of $F$-convergence of sequences. It is clear that $x-y \in \mathcal{N}_{\theta, z}$ means $y \in \mathcal{N}_{x, z}$ and vice versa.

A sequence $x=\left(x_{n}\right)$ in $X$ is said to be $F$-convergence to $L \in X$ if for every $\varepsilon>0, \eta \in(0,1)$ and for each nonzero $z \in X$ there exists a positive integer $N$ such that

$$
x_{n}, z-L \in \mathcal{N}_{\theta, z}(\varepsilon, \eta) \text { for each } n \geq N
$$

or equivalently,

$$
x_{n}, z \in \mathcal{N}_{L, z}(\varepsilon, \eta) \text { for each } n \geq N .
$$

In this case we write $F$ - $\lim x_{n}, z=L$.

\section{The Main Results}

It is known (see [16]) that statistical cluster $\Gamma_{x}$ and statistical limit points set $\Lambda_{x}$ of a given sequence $\left(x_{n}\right)$ are not altered by changing the values of a subsequence, the index set of which has density zero. Moreover, there is a strong connection between $\theta$-statistical cluster points and ordinary limit points of a given sequence. We will prove that these facts are satisfied for $\theta$-statistical cluster points and $\theta$-statistical limit point sets of a given sequence in the topology induced by random 2-normed spaces.

The notion of statistical convergence depends on the density of subsets of $\mathbb{N}$, the set of natural numbers.

Definition 2 (see $[8,11]$ ). Let $K$ be a subset of $\mathbb{N}$. Then the asymptotic density of $K$ denoted by $\delta(K)$ := $\lim _{n \rightarrow \infty}(1 / n)|\{k \leq n: k \in K\}|$, where the vertical bars denote the cardinality of the enclosed set. A number sequence $x=\left(x_{k}\right)_{k \in \mathbb{N}}$ is said to be statistically convergent to $L$ if for every $\varepsilon>0, \delta\left(\left\{k \in \mathbb{N}:\left|x_{k}-L\right| \geq \varepsilon\right\}\right)=0$. If $\left(x_{k}\right)_{k \in \mathbb{N}}$ is statistically convergent to $L$, we write $s t-\lim x_{k}=L$.

By a lacunary sequence we mean an increasing integer sequence $\theta=\left(k_{r}\right)$ such that $k_{0}=0$ and $h_{r}:=k_{r}-k_{r-1} \rightarrow \infty$ as $r \rightarrow \infty$. Throughout this paper the intervals determined by $\theta$ will be denoted by $I_{r}:=\left(k_{r-1}, k_{r}\right]$, and the ratio $k_{r} / k_{r-1}$ will be abbreviated by $q_{r}$. Let $K \subseteq \mathbb{N}$. The number

$$
\delta_{\theta}(K)=\lim _{r} \frac{1}{h_{r}}\left|\left\{k \in I_{r}: k \in K\right\}\right|
$$

is said to be the $\theta$-density of $K$, provided the limit exists (see [17]).

Definition 3 (see [17]). Let $\theta$ be a lacunary sequence. Then a sequence $x=\left(x_{k}\right)$ is said to be $S_{\theta}$-convergent to the number $L$ if for every $\varepsilon>0$ the set $K(\varepsilon)$ has $\theta$-density zero, where

$$
K(\varepsilon):=\left\{k \in \mathbb{N}:\left|x_{k}-L\right| \geq \varepsilon\right\} .
$$

In this case we write $S_{\theta}-\lim x=L$ or $x_{k} \rightarrow L\left(S_{\theta}\right)$.

Definition 4 (see [12]). Let $(X, F, *)$ be a RTN space and let $\theta$ be a lacunary sequence. A sequence $x=\left(x_{k}\right)$ in a random 2-normed spaces $(X, F, *)$ is said to be $\theta$-statistically convergent or $S_{\theta}$-convergent to $L \in X$ with respect to $F$ if for every $\varepsilon>0, \eta \in(0,1)$ and nonzero $z \in X$ such that

$$
\delta_{\theta}\left(\left\{k \in \mathbb{N}: x_{k}, z \notin \mathcal{N}_{L, z}(\varepsilon, \eta)\right\}\right)=0
$$

or equivalently

$$
\delta_{\theta}\left(\left\{k \in \mathbb{N}: x_{k}, z \in \mathcal{N}_{L, z}(\varepsilon, \eta)\right\}\right)=1 .
$$

In this case we write $S_{\theta}^{\mathrm{RTN}}-\lim x, z=L$ or $x_{k} \rightarrow L\left(S_{\theta}^{\mathrm{RTN}}\right)$.

Now we define some concepts in RTN-space.

Definition 5. Let $(X, F, *)$ be a RTN space and let $\theta$ be a lacunary sequence. Let $\left(x_{k(j)}\right)$ be a subsequence of $x=\left(x_{k}\right) \in$ $X$ and $K=\{k(j): j \in \mathbb{N}\}$ then one denotes $\left(x_{k(j)}\right)$ by $(x)_{K}$. If

$$
\lim _{r} \frac{1}{h_{r}}\left|\left\{k(j) \in I_{r}: j \in \mathbb{N}\right\}\right|=0,
$$


then $(x)_{K}$ is called a $\theta$-thin sequence. On the other hand, $(x)_{K}$ is a $\theta$-nonthin subsequence of $x$ provided that

$$
\lim \sup _{r} \frac{1}{h_{r}}\left|\left\{k(j) \in I_{r}: j \in \mathbb{N}\right\}\right|>0 .
$$

Definition 6. Let $(X, F, *)$ be a RTN space and $\theta$ be a lacunary sequence. $A \in X$ is called a $\theta$-statistical limit point of a sequence $x=\left(x_{k}\right) \in X$ provided that there is a $\theta$-nonthin subsequence of $x$ that converges to $A$. Let $\Lambda_{\theta, x}^{\mathrm{RTN}}$ denotes the set of all $S_{\theta}^{\mathrm{RTN}}$-limit points.

Definition 7. Let $(X, F, *)$ be a RTN space and let $\theta$ be a lacunary sequence. $B \in X$ is called a $\theta$-statistical cluster point of a sequence $x=\left(x_{k}\right) \in X$ provided that, for every $\varepsilon>0$, $\eta \in(0,1)$ and nonzero $z \in X$

$$
\lim \sup _{r} \frac{1}{h_{r}}\left|\left\{k \in I_{r}: x_{k}, z \in \mathcal{N}_{L, z}(\varepsilon, \eta)\right\}\right|>0 .
$$

Let $\Gamma_{\theta, x}^{\mathrm{RTN}}$ denote the set of $\theta$-statistical cluster point of the sequence $x=\left(x_{k}\right)$.

Theorem 8. Let $(X, F, *)$ be a RTN space and let $\theta$ be a lacunary sequence. If $x=\left(x_{k}\right)$ and $y=\left(y_{k}\right)$ are sequences in $X$ such that

$$
\lim _{r} \frac{1}{h_{r}}\left|\left\{k \in I_{r}: x_{k} \neq y_{k}\right\}\right|=0,
$$

then $\Lambda_{\theta, x}^{R T N}=\Lambda_{\theta, y}^{R T N}$ and $\Gamma_{\theta, x}^{R T N}=\Gamma_{\theta, y}^{R T N}$.

Proof. Assume that $\lim _{r}\left(1 / h_{r}\right)\left|\left\{k \in I_{r}: x_{k} \neq y_{k}\right\}\right|=0$ and $A \epsilon$ $\Lambda_{\theta, y}^{\text {RTN }}$; say $(y)_{K}$ is a $\theta$-nonthin sequence of $y$ that converges to A. Since

$$
\lim _{r} \frac{1}{h_{r}} \mid\left\{k \in I_{r}: k \in K \text { and } x_{k} \neq y_{k}\right\} \mid=0,
$$

it follows that

$$
\lim \sup _{r} \frac{1}{h_{r}} \mid\left\{k \in I_{r}: k \in K \text { and } x_{k}=y_{k}\right\} \mid \neq 0 .
$$

Therefore, the latter set yields a $\theta$-nonthin subsequence $(x)_{K^{\prime}}$ of $(x)_{K}$ that converges to $A$. Hence $A \in \Lambda_{\theta, x}^{\mathrm{RTN}}$ and $\Lambda_{\theta, y}^{\mathrm{RTN}} \subseteq$ $\Lambda_{\theta, x}^{\mathrm{RTN}}$. By symmetry we see that $\Lambda_{\theta, x}^{\mathrm{RTN}} \subseteq \Lambda_{\theta, y}^{\mathrm{RTN}}$; hence $\Lambda_{\theta, x}^{\mathrm{RTN}}=$ $\Lambda_{\theta, y}^{\mathrm{RTN}}$. Now let $B \in \Gamma_{\theta, x}^{\mathrm{RTN}}$ and $\operatorname{let}_{\lim _{r}}\left(1 / h_{r}\right)\left|\left\{k \in I_{r}: x_{k} \neq y_{k}\right\}\right|=$ 0 . Since $B \in \Gamma_{\theta, x}^{\mathrm{RTN}}$, we can write

$$
\lim \sup _{r} \frac{1}{h_{r}}\left|\left\{k \in I_{r}: x_{k}, z \in \mathcal{N}_{B, z}(\varepsilon, \eta)\right\}\right| \neq 0
$$

for every $\varepsilon>0, \eta \in(0,1)$, and nonzero $z \in X$. Since $x_{k}=y_{k}$ for almost all $k$,

$$
\lim \sup _{r} \frac{1}{h_{r}}\left|\left\{k \in I_{r}: y_{k}, z \in \mathcal{N}_{B, z}(\varepsilon, \eta)\right\}\right| \neq 0
$$

for every $\varepsilon>0, \eta \in(0,1)$, and nonzero $z \in X$. Hence, $B \in$ $\Gamma_{\theta, y}^{\mathrm{RTN}}$ and $\Gamma_{\theta, x}^{\mathrm{RTN}} \subseteq \Gamma_{\theta, y}^{\mathrm{RTN}}$. By symmetry we see that $\Gamma_{\theta, y}^{\mathrm{RTN}} \subseteq \Gamma_{\theta, x}^{\mathrm{RTN}}$; hence $\Gamma_{\theta, x}^{\mathrm{RTN}}=\Gamma_{\theta, y}^{\mathrm{RTN}}$.
Theorem 9. Let $(X, F, *)$ be a RTN space and let $\theta$ be a lacunary sequence. For any sequence $x=\left(x_{k}\right) \in X$, one has $\Lambda_{\theta, x}^{R T N} \subseteq \Gamma_{\theta, x}^{R T N}$.

Proof. Suppose $A \in \Lambda_{\theta, x}^{\mathrm{RTN}}$; then there is a $\theta$-nonthin subsequence $\left(x_{k(j)}\right)$ of $x$ that converges to $A$, that is,

$$
\lim \sup _{r} \frac{1}{h_{r}}\left|\left\{k(j) \in I_{r}: j \in \mathbb{N}\right\}\right|=d>0 .
$$

Since

$$
\begin{aligned}
\{k & \left.\in \mathbb{N}: x_{k}, z \in \mathcal{N}_{A, z}(\varepsilon, \eta)\right\} \\
& \supseteq\left\{k(j) \in \mathbb{N}: x_{k(j)}, z \in \mathcal{N}_{A, z}(\varepsilon, \eta)\right\}
\end{aligned}
$$

for every $\varepsilon>0, \eta \in(0,1)$, and nonzero $z \in X$, we have

$$
\begin{aligned}
& \left\{k \in \mathbb{N}: x_{k}, z \in \mathcal{N}_{A, z}(\varepsilon, \eta)\right\} \\
& \quad \supseteq\{k(j) \in \mathbb{N}: j \in \mathbb{N}\} \backslash\left\{k(j) \in \mathbb{N}: x_{k(j)}, z \notin \mathcal{N}_{A, z}(\varepsilon, \eta)\right\} .
\end{aligned}
$$

Since $\left(x_{k(j)}\right)$ converges to $A$, the set

$$
\left\{k(j) \in \mathbb{N}: x_{k(j)}, z \notin \mathcal{N}_{A, z}(\varepsilon, \eta)\right\}
$$

is finite for any $\varepsilon>0, \eta \in(0,1)$, and nonzero $z \in X$. Therefore,

$$
\begin{aligned}
& \lim \sup _{r} \frac{1}{h_{r}}\left|\left\{k(j) \in I_{r}: x_{k}, z \in \mathcal{N}_{A, z}(\varepsilon, \eta)\right\}\right| \\
& \geq \lim \sup _{r} \frac{1}{h_{r}}\left|\left\{k(j) \in I_{r}: j \in \mathbb{N}\right\}\right| \\
& \quad-\lim \sup _{r} \frac{1}{h_{r}}\left|\left\{k(j) \in I_{r}: x_{k(j)}, z \notin \mathcal{N}_{A, z}(\varepsilon, \eta)\right\}\right| \\
& \geq \lim _{r} \frac{1}{h_{r}}\left|\left\{k(j) \in I_{r}: j \in \mathbb{N}\right\}\right|=d>0 .
\end{aligned}
$$

Hence,

$$
\lim \sup _{r} \frac{1}{h_{r}}\left|\left\{k(j) \in I_{r}: x_{k}, z \in \mathcal{N}_{A, z}(\varepsilon, \eta)\right\}\right|>0
$$

which means that $A \in \Gamma_{\theta, x}^{\mathrm{RTN}}$.

Theorem 10. Let $(X, F, *)$ be a RTN space and let $\theta$ be a lacunary sequence. Let $L_{x}^{R T N}$ be the set of ordinary limit points of $x$ and for any sequence $x=\left(x_{k}\right) \in X, \Gamma_{\theta, x}^{R T N} \subseteq L_{x}^{R T N}$.

Proof. Assume that $B \in \Gamma_{\theta, x}^{\mathrm{RTN}}$; then

$$
\lim \sup _{r} \frac{1}{h_{r}}\left|\left\{k \in I_{r}: x_{k}, z \in \mathcal{N}_{B, z}(\varepsilon, \eta)\right\}\right|>0
$$

for every $\varepsilon>0, \eta \in(0,1)$, and nonzero $z \in X$. We set $(x)_{K}$ a $\theta$-nonthin subsequence of $x$ such that

$$
K=\left\{k(j) \in \mathbb{N}: x_{k(j)}, z \in \mathcal{N}_{B, z}(\varepsilon, \eta)\right\}
$$


for every $\varepsilon>0, \eta \in(0,1)$, and nonzero $z \in X$, and $\lim \sup _{r}\left(1 / h_{r}\right)|K|>0$. Since there are infinitely many elements in $K, B \in L_{x}^{\mathrm{RTN}}$.

The converse of the theorem does not hold.

Theorem 11. Let $(X, F, *)$ be a RTN space and let $\theta$ be a lacunary sequence. If for sequence $x=\left(x_{k}\right) \in X, S_{\theta}^{R T N}-\lim x_{k}=x_{0}$, then $\Lambda_{\theta, x}^{R T N}=\Gamma_{\theta, x}^{R T N}=\left\{x_{0}\right\}$.

Proof. First, we show that $\Lambda_{\theta, x}^{\mathrm{RTN}}=\left\{x_{0}\right\}$. Fix $\varepsilon>0, \eta \in(0,1)$, and nonzero $z \in X$. Assume that $\Lambda_{\theta, x}^{\mathrm{RTN}}=\left\{x_{0}, y_{0}\right\}$ such that $x_{0}, z \notin \mathcal{N}_{y_{0}, z}(2 \varepsilon, \eta)$. In this case, there exist $\left(x_{k(j)}\right)$ and $\left(x_{l(i)}\right) \theta$-nonthin subsequences of $x=\left(x_{k}\right)$ that converge to $x_{0}$ and $y_{0}$, respectively. Since $\left(x_{l(i)}\right)$ converges to $y_{0}$, we have

$$
\left\{l(i) \in \mathbb{N}: x_{l(i)}, z \notin \mathcal{N}_{y_{0}, z}(\varepsilon, \eta)\right\}
$$

which is a finite set. Consider that

$$
\begin{aligned}
\{l(i) & \in \mathbb{N}: i \in \mathbb{N}\} \\
= & \left\{l(i) \in \mathbb{N}: x_{l(i)}, z \in \mathcal{N}_{y_{0}, z}(\varepsilon, \eta)\right\} \\
& \cup\left\{l(i) \in \mathbb{N}: x_{l(i)}, z \notin \mathcal{N}_{y_{0}, z}(\varepsilon, \eta)\right\}
\end{aligned}
$$

implies

$$
\begin{aligned}
& \lim \sup _{r} \frac{1}{h_{r}}\left|\left\{l(i) \in I_{r}: i \in \mathbb{N}\right\}\right| \\
& =\lim \sup _{r} \frac{1}{h_{r}}\left|\left\{l(i) \in I_{r}: x_{l(i)}, z \in \mathcal{N}_{y_{0}, z}(\varepsilon, \eta)\right\}\right| \\
& \quad+\lim \sup _{r} \frac{1}{h_{r}}\left|\left\{l(i) \in I_{r}: x_{l(i)}, z \notin \mathcal{N}_{y_{0}, z}(\varepsilon, \eta)\right\}\right| .
\end{aligned}
$$

Hence,

$$
\lim \sup _{r} \frac{1}{h_{r}}\left|\left\{l(i) \in I_{r}: x_{l(i)}, z \in \mathcal{N}_{y_{0}, z}(\varepsilon, \eta)\right\}\right|>0 .
$$

Since $S_{\theta}^{\mathrm{RTN}}-\lim x_{k}=x_{0}$,

$$
\lim _{r} \frac{1}{h_{r}}\left|\left\{k \in I_{r}: x_{k}, z \notin \mathcal{N}_{x_{0}, z}(\varepsilon, \eta)\right\}\right|=0 .
$$

Therefore, we can write

$$
\lim \sup _{r} \frac{1}{h_{r}}\left|\left\{k \in I_{r}: x_{k}, z \in \mathcal{N}_{x_{0}, z}(\varepsilon, \eta)\right\}\right|>0 .
$$

For every $x_{0}, z \notin \mathcal{N}_{y_{0}, z}(2 \varepsilon, \eta)>0$,

$$
\begin{aligned}
& \left\{l(i) \in \mathbb{N}: x_{l(i)}, z \in \mathcal{N}_{y_{0}, z}(\varepsilon, \eta)\right\} \\
& \cap\left\{k \in \mathbb{N}: x_{k}, z \in \mathcal{N}_{x_{0}, z}(\varepsilon, \eta)\right\}=\emptyset .
\end{aligned}
$$

Hence,

$$
\begin{aligned}
& \left\{l(i) \in \mathbb{N}: x_{l(i)}, z \in \mathcal{N}_{y_{0}, z}(\varepsilon, \eta)\right\} \\
& \subseteq\left\{k \in \mathbb{N}: x_{k}, z \notin \mathcal{N}_{x_{0}, z}(\varepsilon, \eta)\right\} .
\end{aligned}
$$

Therefore,

$$
\begin{aligned}
& \lim \sup _{r} \frac{1}{h_{r}}\left|\left\{l(i) \in I_{r}: x_{l(i)}, z \in \mathcal{N}_{y_{0}, z}(\varepsilon, \eta)\right\}\right| \\
& \leq \lim \sup _{r} \frac{1}{h_{r}}\left|\left\{k \in I_{r}: x_{k}, z \notin \mathcal{N}_{x_{0}, z}(\varepsilon, \eta)\right\}\right|=0 .
\end{aligned}
$$

This contradicts (31). Hence, $\Lambda_{\theta, x}^{\mathrm{RTN}}=\left\{x_{0}\right\}$.

Now we assume that $\Gamma_{\theta, x}^{\mathrm{RTN}}=\left\{x_{0}, z_{0}\right\}$ such that $x_{0}, z \notin$ $\mathcal{N}_{z_{0}, z}(2 \varepsilon, \eta)$ for some $\varepsilon>0, \eta \in(0,1)$, and nonzero $z \in X$. Then

$$
\lim \sup _{r} \frac{1}{h_{r}}\left|\left\{k \in I_{r}: x_{k}, z \in \mathcal{N}_{z_{0}, z}(\varepsilon, \eta)\right\}\right|>0 .
$$

Since

$$
\begin{aligned}
& \left\{k \in \mathbb{N}: x_{k}, z \in \mathcal{N}_{x_{0}, z}(\varepsilon, \eta)\right\} \\
& \quad \cap\left\{k \in \mathbb{N}: x_{k}, z \in \mathcal{N}_{z_{0}, z}(\varepsilon, \eta)\right\}=\emptyset,
\end{aligned}
$$

for every $x_{0}, z \notin \mathcal{N}_{z_{0}, z}(2 \varepsilon, \eta)$,

$$
\left\{k \in \mathbb{N}: x_{k}, z \notin \mathcal{N}_{x_{0}, z}(\varepsilon, \eta)\right\} \supseteq\left\{k \in \mathbb{N}: x_{k}, z \in \mathcal{N}_{z_{0}, z}(\varepsilon, \eta)\right\} .
$$

Therefore

$$
\begin{aligned}
& \lim \sup _{r} \frac{1}{h_{r}}\left|\left\{k \in I_{r}: x_{k}, z \notin \mathcal{N}_{x_{0}, z}(\varepsilon, \eta)\right\}\right| \\
& \geq \lim \sup _{r} \frac{1}{h_{r}}\left|\left\{k \in I_{r}: x_{k}, z \in \mathcal{N}_{z_{0}, z}(\varepsilon, \eta)\right\}\right| .
\end{aligned}
$$

From (37), the right side of (40) is greater than zero and from (32), the left side of (40) equals to zero. This is a contradiction. Hence, $\Gamma_{\theta, x}^{\mathrm{RTN}}=\left\{x_{0}\right\}$.

\section{References}

[1] K. Menger, "Statistical metrics," Proceedings of the National Academy of Sciences of the United States of America, vol. 28, pp. 535-537, 1942.

[2] B. Schweizer and A. Sklar, Probabilistic Metric Spaces, NorthHolland, New York, NY, USA, 1983.

[3] I. Goleț, "On probabilistic 2-normed spaces," Novi Sad Journal of Mathematics, vol. 35, no. 1, pp. 95-102, 2005.

[4] S. Gähler, "2-metrische Räume und ihre topologische Struktur," Mathematische Nachrichten, vol. 26, pp. 115-148, 1963.

[5] M. Mursaleen and S. A. Mohiuddine, "On ideal convergence of double sequences in probabilistic normed spaces," Mathematical Reports, vol. 12, no. 4, pp. 359-371, 2010.

[6] M. Mursaleen, "On statistical convergence in random 2-normed spaces," Acta Scientiarum Mathematicarum, vol. 76, no. 1-2, pp. 101-109, 2010.

[7] M. Mursaleen and S. A. Mohiuddine, "On ideal convergence in probabilistic normed spaces," Mathematica Slovaca, vol. 62, no. 1, pp. 49-62, 2012. 
[8] H. Fast, "Sur la convergence statistique," Colloquium Mathematicae, vol. 2, pp. 241-244, 1951.

[9] H. Steinhaus, "Sur la convergence ordinaire et la convergence asymptotique," Colloquium Mathematicum, vol. 2, pp. 73-74, 1951.

[10] T. Salat, "On statistical convergence of real numbers," Mathematica Slovaca, vol. 50, pp. 111-115, 2000.

[11] J. A. Fridy, “On statistical convergence," Analysis, vol. 5, no. 4, pp. 301-313, 1985.

[12] S. A. Mohiuddine and M. Aiyub, "Lacunary statistical convergence in random 2-normed spaces," Applied Mathematics \& Information Sciences, vol. 6, no. 3, pp. 581-585, 2012.

[13] M. Mursaleen and S. A. Mohiuddine, "On lacunary statistical convergence with respect to the intuitionistic fuzzy normed space," Journal of Computational and Applied Mathematics, vol. 233, no. 2, pp. 142-149, 2009.

[14] S. A. Mohiuddine and E. Savaş, "Lacunary statistically convergent double sequences in probabilistic normed spaces," Annali dell'Universitá di Ferrara, vol. 58, no. 2, pp. 331-339, 2012.

[15] S. Gähler, "Lineare 2-normierte Räume," Mathematische Nachrichten, vol. 28, pp. 1-43, 1964.

[16] J. A. Fridy, "Statistical limit points," Proceedings of the American Mathematical Society, vol. 118, no. 4, pp. 1187-1192, 1993.

[17] J. A. Fridy and C. Orhan, "Lacunary statistical convergence," Pacific Journal of Mathematics, vol. 160, no. 1, pp. 43-51, 1993. 


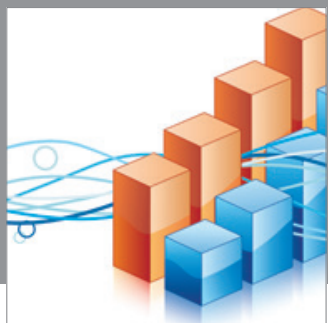

Advances in

Operations Research

mansans

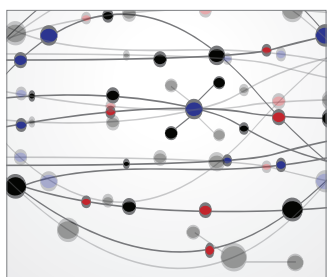

The Scientific World Journal
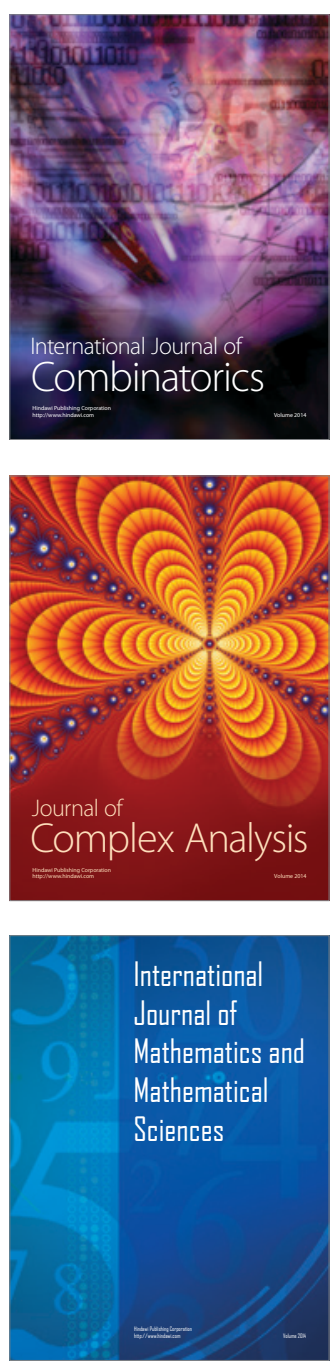
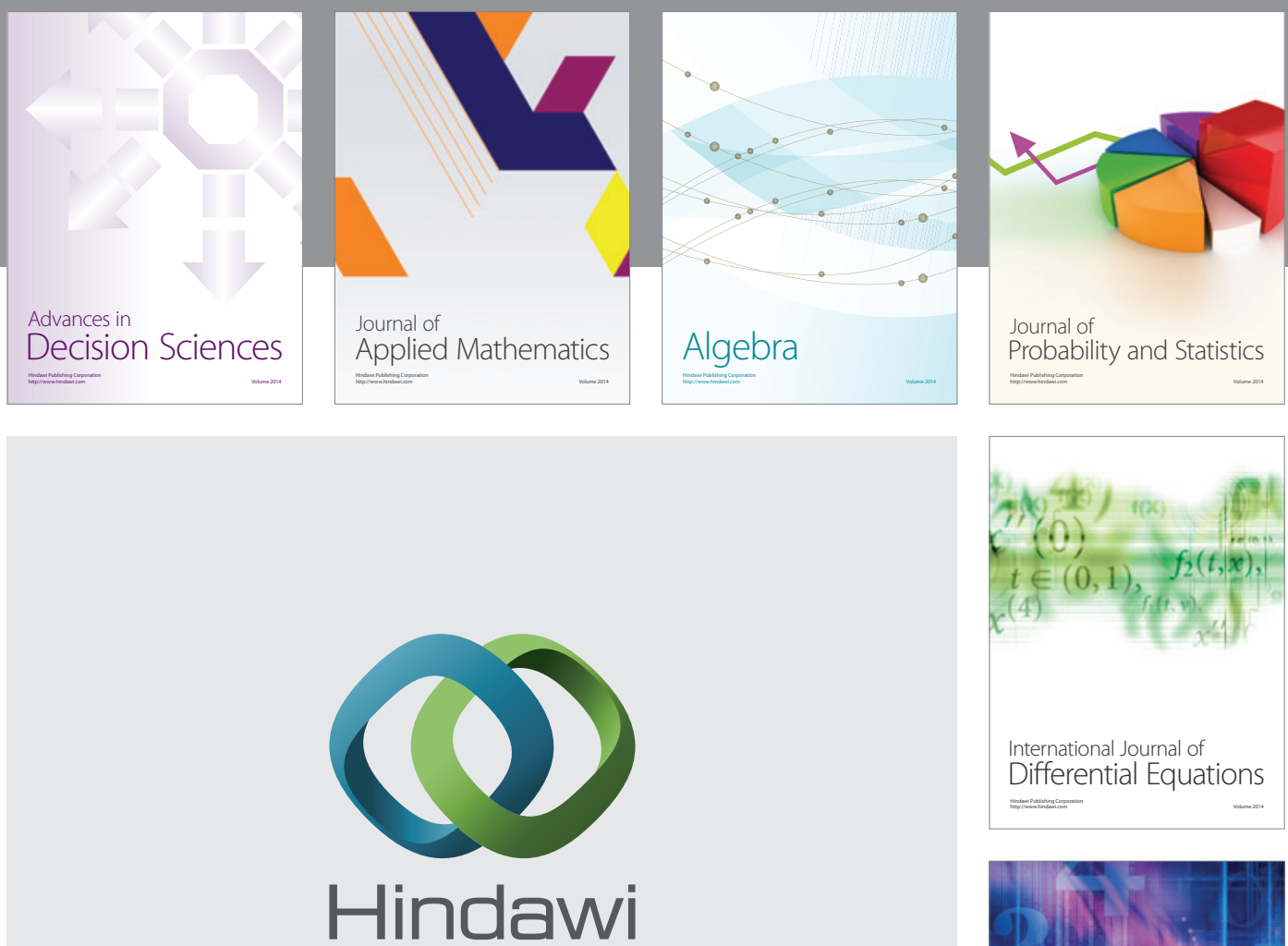

Submit your manuscripts at http://www.hindawi.com
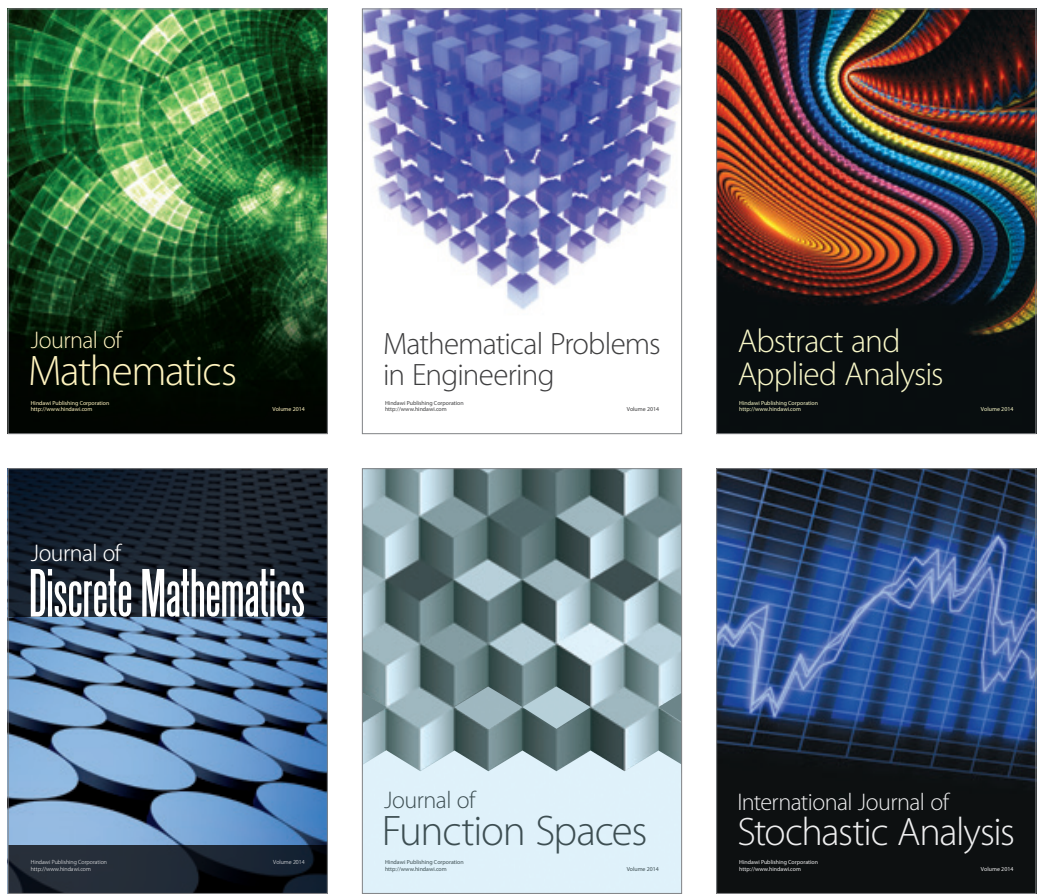

Journal of

Function Spaces

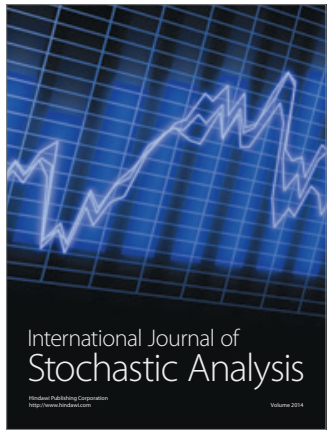

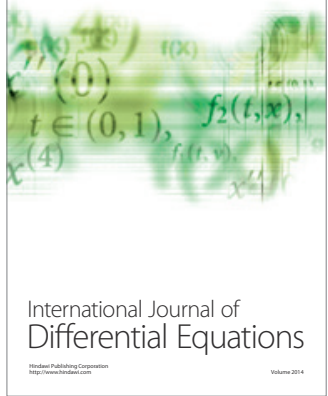
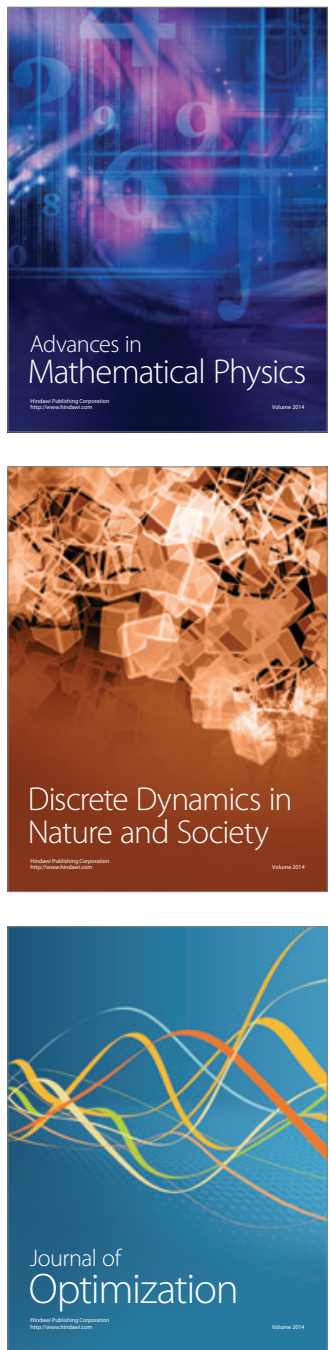